\title{
Autoimmunity: Basic Mechanisms and Implications in Endocrine Diseases
}

\section{Part I}

\author{
S. Ballotti ${ }^{\mathrm{a}} \quad$ F. Chiarelli ${ }^{\mathrm{b}} \quad$ M. de Martino ${ }^{\mathrm{a}}$ \\ a Department of Paediatrics, Anna Meyer Children's Hospital, University of Florence, Florence, and \\ ${ }^{b}$ Department of Paediatrics, University of Chieti, Chieti, Italy
}

\section{Key Words}

Central tolerance $\cdot \mathrm{HLA}$-associated diseases ·

Epigenetic mechanisms · Negative selection •

Molecular mimicry $\cdot$ Apoptosis

\begin{abstract}
Autoimmunity implies disturbances at several levels of the immune control. Self-tolerance and discrimination between self and non-self synergize to avoid the development of autoimmunity. Negative selection in the thymus, the transcription factor AIRE, CD4+CD25+ regulatory T cells, and dendritic cells cooperate to produce and maintain tolerance. Cytokines modulate deriving immune processes and influence the local micro-environment. Multiple mechanisms are involved in tolerance breakdown: genetic factors (major histocompatibility complex haplotypes, polymorphisms in the cytotoxic T lymphocyte antigen gene and epigenetic alterations), environmental factors (mainly infections), impaired apoptosis, and the emergence of autoreactive naive lymphocytes. These events may be involved in the pathogenesis of endocrine diseases at several levels.
\end{abstract}

Copyright $\odot 2006$ S. Karger AG, Basel
(C) 2006 S. Karger AG, Basel

0301-0163/06/0663-0132\$23.50/0

Fax +4161306 1234

E-Mail karger@karger.ch

www.karger.com
Accessible online at:

www.karger.com/hre

\section{Introduction}

Discrimination between self- and non-self-antigens is a complex process that implies the preservation of selftolerance and the enhancement of the ability to assemble an effective immune response. Several factors are involved in the initiation of autoimmunity, including genetic predisposition, exposure to environmental factors and alterations in mechanisms of central and peripheral tolerance $[1,2]$.

In this first part, we will analyze the genetic mechanisms underlying autoimmunity, the role of environmental factors, and the significance of apoptotic processes.

\section{HLA-Associated Diseases: The Type 1 \\ Diabetes Mellitus Model}

A critical role in enhancing the autoimmunity risk is played by major histocompatibility complex (MHC) haplotypes.

MHC is a complex of genes with a high degree of polymorphism whose products are expressed on the surface of several cellular types. Because of their rich expression on the membrane of leukocytes, these molecules were also called human leukocyte antigens (HLAs). The HLA system is located on chromosome 6 , and the whole allelic set on each chromosome is indicated as MHC haplotype

Prof. Maurizio de Martino, MD

Department of Paediatrics, University of Florence

via Luca Giordano, 13

IT-50132 Florence (Italy)

Tel. +39 0555662 494, Fax +39 0555 70380,E-Mail maurizio.demartino@unifi.it 
(fig. 1). It is possible that alleles from different loci are inherited in association (linkage disequilibrium). Similar haplotypes are often correlated with autoimmune diseases [3-5].

T lymphocytes are able to recognize specific antigens only when they are associated with MHC molecules on the cellular surface (fig. 2). Polymorphisms are fundamental to diversify the structure of MHC molecules and consequently allow the interaction with different processed antigens. Polymorphisms are also present in the binding site with $\mathrm{T}$ lymphocytes. This may condition a diverse binding affinity and increase the antigen presentation in the periphery, with a consequent enhanced $\mathrm{T}$ cell stimulation [6].

The T cell capacity to interact with MHC molecules is also critical for their positive selection. Indeed, in the thymus, the $\mathrm{CD} 4+\mathrm{CD} 8+$ (double-positive, $\mathrm{DP}$ ) thymocytes, derived from bone marrow hemopoietic precursors, interact with cortical epithelial cells, which express a high level of MHC class I and class II molecules associated with self-peptides. DP cells bearing the $\mathrm{T}$ cell receptor (TCR) with appropriate affinity for peptide-MHC complexes are positively selected, thus ensuring that all mature T cells have some affinity for MHC molecules (MHC restriction). Thymocytes expressing TCRs that interact with peptide-MHC class I complexes become CD8+ T cells, while those that express TCRs binding peptideMHC class II complexes become CD4+ T cells (fig. 3 ). However, peripheral DP T cells have been demonstrated in several pathological conditions and in normal individuals $[7,8]$.

Type 1 diabetes mellitus (T1D) represents an interesting model of HLA-associated disease. A recent review by Mimi and Polychronakos [9] analyzed the correlation between HLA haplotypes and the risk for T1D. Polymorphisms in HLA DRB, HLA DQB and HLA DQA genes appear to be critical in T1D, where polymorphisms in the insulin gene seem to be the strongest candidate for insulin-dependent diabetes mellitus type 2 (IDDM2). HLA alleles may be markers for T1D susceptibility but they do not themselves represent the susceptibility genes, which are still unknown [9-12].

T1D susceptibility displays unusual patterns of inheritance. The analysis of pairs of affected sibs and their MHC haplotypes suggests a recessive inheritance for some susceptibility genes, while protective MHC alleles may express a dominant nature $[12,13]$. This difference seems correlated to the binding competition for $\beta$-cell antigens among MHC molecules. A MHC molecule with a high affinity for a diabetogenic antigen binds it prefer- entially and expresses a dominant nature. Conversely, if the MHC haplotype encodes for a molecule with autoantigen low affinity, a high number of molecules will be necessary to compete efficiently and this is achieved in a homozygous state $[13,14]$. Furthermore, there are parental differences in T1D risk factors, so the offspring risk is higher if the father is affected, especially if diabetes has appeared at an early age [13]. There may be several explanations for this phenomenon: a genetic mechanism similar to imprinting, an environmental protective factor active during pregnancy, or the lower genetic risk associated with mothers who develop the disease at a later age $[13,14]$.

Furthermore, disease expression is sometimes different in genetically identical patients, probably because of the 'incomplete penetrance'. Two different mechanisms have been proposed to explain this phenomenon: an environmental trigger, such as viral infections, drugs or other chemical exposure, and an intrinsic mechanism, such as epigenetic alterations in genomic DNA [15]. Similar patterns are present in IgA and IgD deficiency, systemic lupus erythematosus (SLE) and celiac disease, other examples of MHC-determined diseases [4].

\section{Polymorphisms of the Cytotoxic T Lymphocyte Antigen 4 Gene}

A recent advance in understanding autoimmune disease susceptibility is correlated with the identification of polymorphisms of the cytotoxic T lymphocyte antigen 4 (CTLA4) gene. CTLA4 is a molecule expressed on the activated $\mathrm{T}$ cell surface, where it encodes for a negative signal that limits T cell responses. Graves' disease, autoimmune hypothyroidism and T1D have been associated with the same region on chromosome $2 q 33$, which includes the T-lymphocyte regulatory genes CD28, CTLA4 and inducible co-stimulator (ICOS) [16, 17]. Ueda et al. [17] analyzed 384 cases of Graves' disease and 652 controls to determine their allele frequencies and identify an association with disease. They evidenced single nucleotide polymorphisms in the 6.1-kb region, which contains most of the CTLA4 3'-untranslated regions. These polymorphisms were correlated to a lower soluble CTLA4 mRNA, in susceptible individuals. So allelic variation of the 6.1-kb region may cause an alteration in splicing efficiency, with a reduction in soluble CTLA4 production [17]. Variations in soluble CTLA4 levels may explain the correlation between CTLA4 gene polymorphisms and the reduction of this inhibitory function, which promotes 
an alteration in $\mathrm{T}$ cell activation in vitro [17]. The G49 allele of CTLA4 has been associated with increased thyroid autoantibody production, above all autoantibodies against thyroid peroxidase and thyroglobulin. However, at present there is still no evidence of a specific correlation between this allele and Graves' disease or Hashimoto thyroiditis. Population genetic studies confirm a critical role for the CTLA4 region in susceptibility to autoimmune thyroid diseases (a genetic linkage between Graves' disease and CTLA4 has been demonstrated in the British population). CTLA4 is probably not a unique determinant and further genetic and functional experiments should be made to evaluate its significance. This may also contribute to elucidate the treatment effects of CTLA4-Ig, a new immunosuppressive therapeutic agent, used in organ transplantation and in autoimmune disease to induce tolerance [18].

\section{Epigenetic Alterations in DNA Sequences and Human Endogenous Retroviruses: The Model of SLE}

Epigenetic alterations are defined as heritable changes in gene expression not affecting nucleotide sequences, but inducing DNA modifications, such as methylation events $[19,20]$. Methylation is a post-synthetic modification, which leads to a downregulation of gene transcription, through the conversion of a cytosine in methyl-cytosine. A large part of methyl-cytosine residues is presented in cytosine and guanine $(\mathrm{CpG})$ dinucleotides, which are mainly contained in the $5^{\prime}$ region of genes. These regions correspond to the promoter and first exon sequences, so different levels of methylation of these sequences influence transcriptional events $[19,20]$.

Several observations suggest a correlation between methylation and SLE: the T cells of SLE patients exhibit a lower level of DNA methylation compared with normal $\mathrm{T}$ cells, and this may be the cause of their inappropriate activation. The hyperactivation of SLE T cells may be explained with the high surface expression of lymphocyte function-associated antigen-1 (LFA-1; CD11a/CD18). Normally, LFA-1 expression is controlled by methylated promoters. The loss of methylation induces an upregulation of LFA- 1 and a possible inappropriate activation of T cells. An example of this phenomenon is offered by the treatment of T cells with 5-aza-deoxycytidine, an inhibitor of DNA methylation. T cells treated with 5-aza-deoxycytidine exhibit a low degree of methylation and undergo activation by the simple interaction with MHC molecules, even without specific antigen [21].
Furthermore, hypomethylated microbial DNA shows a mitogenic effect on B lymphocytes, while vertebrate DNA does not. Methylation of CpG motifs abrogates this effect. Thus, the DNA methylation level represents a distinguishing element between bacterial and vertebrate DNA. Accelerated apoptosis with defective clearance may determine an elevated level of plasma-circulating nuclear antigen, which contains hypomethylated $\mathrm{CpG}$ rich DNA fragments. These fragments may mimic microbial DNA and induce immune cell activation with anti-ds DNA antibody production and cytokine production, as observed in SLE [20-22]. Also drugs, such as procainamide and hydralazine, are associated with SLE and they are, as well as ultraviolet light, hypomethylation agents.

Human endogenous retroviruses (HERVs) are largely represented in human genome but normally not activated. A high level of HERV clone 4-1 mRNA has been demonstrated in SLE patients compared with normal controls, and this may contribute to the pathogenesis of the disease. In fact, expression of HERV components may enhance the anti-ds DNA antibody production due to the homology between HERV sequences and nuclear antigens or by the activation of $\mathrm{T}$ and $\mathrm{B}$ memory lymphocytes $[23,24]$.

\section{Negative Selection and Autoimmunity: Promiscuous Gene Expression in mTECs, Transcription Factor AIRE and Autoimmune Polyendocrinopathy- Candidiasis-Ectodermal Dystrophy}

Negative selection in the thymus contributes to the deletion of autoreactive $T$ cells, so alterations in this process may promote autoimmunity $[25,26]$.

Insulin and pro-insulin are autoantigens and insulin autoantibodies are important risk factors in siblings of T1D individuals. In mice, two isoforms of pro-insulin exist, and pro-insulin-2 represents the principle isoform targeted during autoimmune reactions. Thébault-Baumont et al. [27] demonstrated that NOD mice, which are deficient for pro-insulin-2, develop accelerated insulitis and diabetes. This may be correlated with a lack in the peripheral $\mathrm{T}$ cell repertoire, due to the absence of pro-insulin-2 within the thymus. Furthermore, allelic variations of pro-insulin correlate with the risk of T1D in humans, while insulin expression within the thymus promotes $\mathrm{T}$ cell tolerance to insulin in mice [27].

Thymic medullary epithelial cells (mTECs) probably play a critical role in central tolerance. mTECs have the 

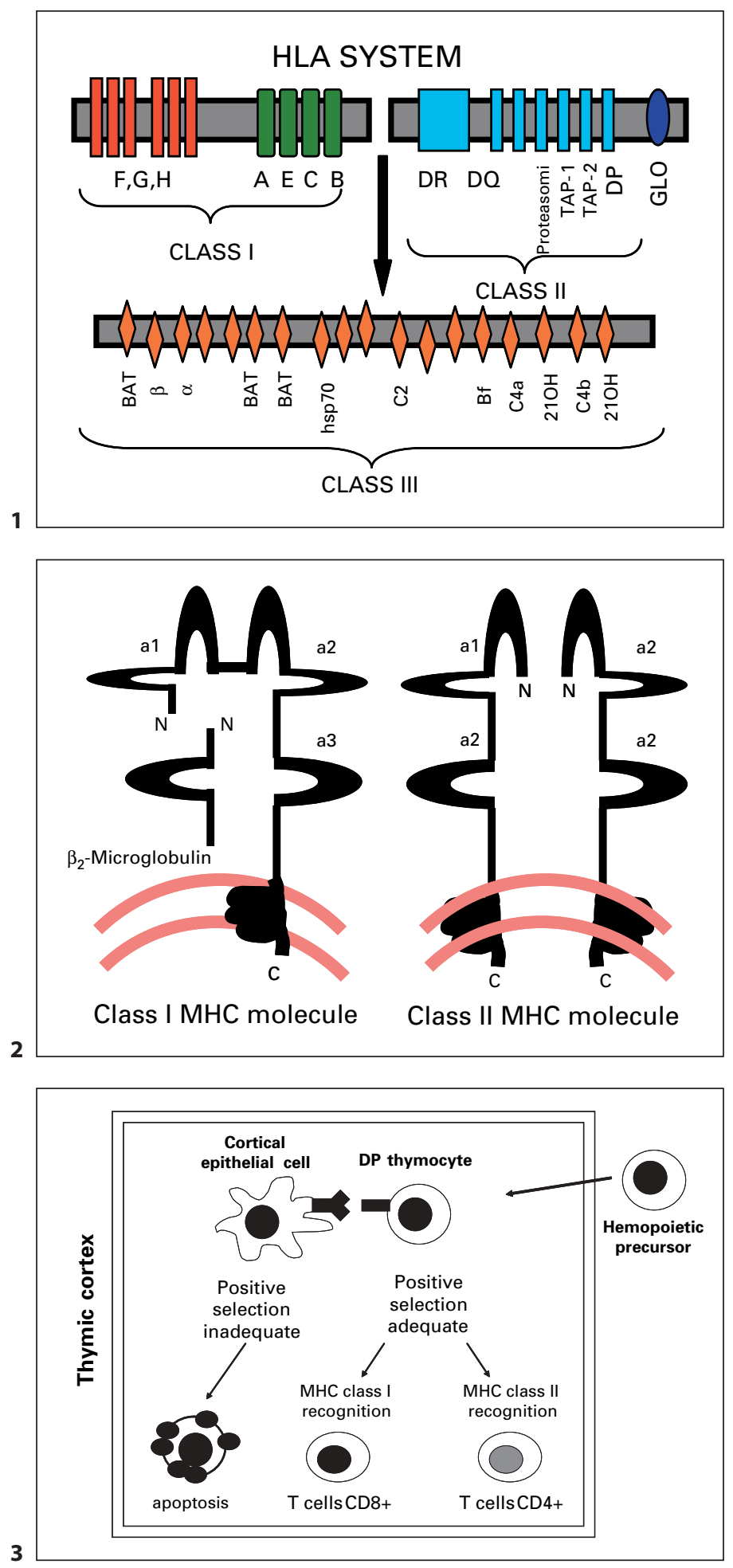

Fig. 1. Schematic distribution of genetic loci of HLA system on chromosome 6.

Fig. 2. Schematic representation of the structure of a class I and class II MHC molecules on the cell surface.

Fig. 3. Positive selection during T-cell development and MHC restriction. In the thymus, the CD4+CD8+ (double-positive, DP)
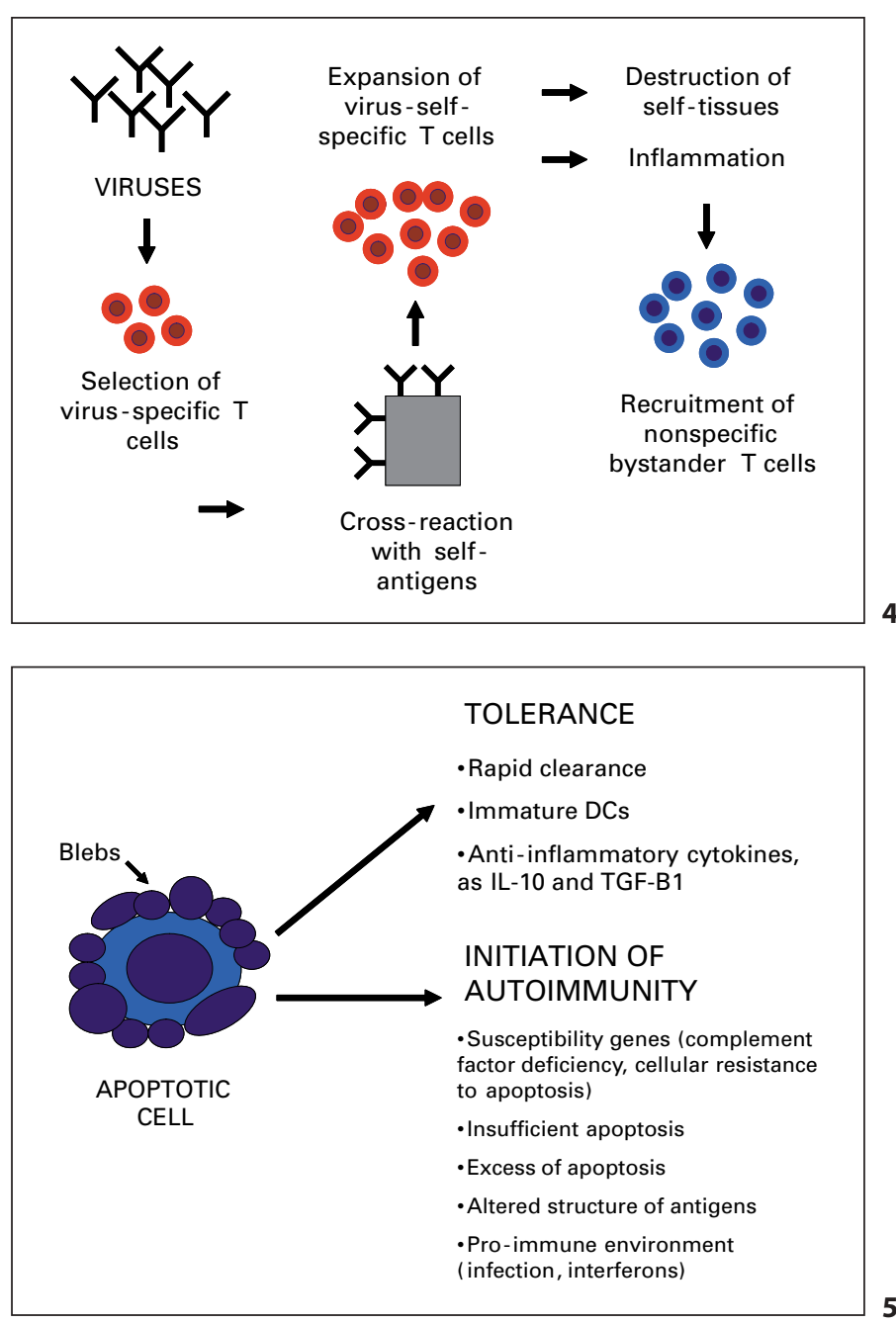

thymocytes derived from bone marrow hemopoietic precursors interact with cortical epithelial cells, expressing a high level of MHC class I and class II molecules associated with self-peptides. DP cells bearing TCR with appropriate affinity for peptide-MHC complexes are positively selected, thus ensuring that all mature $\mathrm{T}$ cells have some affinity for MHC molecules (MHC restriction). Thymocytes expressing TCRs that interact with peptide-MHC class I complexes become CD8+ T cells, while those that express TCRs binding peptide-MHC class II complexes become CD4+ T cells.

Fig. 4. Molecular mimicry may initiate autoimmune events because of cross-reactivity between foreign and self-antigens, with a consequent selection of potential autoreactive $\mathrm{T}$ cells. The initial event may propagate through bystander mechanisms which involve inflammation and cytokine production and lead to the polyclonal activation of $\mathrm{T}$ cells, including low-affinity autoreactive T cells.

Fig. 5. Apoptosis may promote tolerance or, alternatively, initiate autoimmune events. Apoptotic cells are an essential source of tolerogenic materials during normal cellular turnover. However, susceptibility factors may allow apoptotic cells to accumulate in a pro-inflammatory manner, with a consequent alteration in homeostatic balance in favor of autoimmunity. 
ability to present antigens generally expressed outside the thymus, such as insulin and glutamic acid decarboxylase. These antigens are expressed in a tissue-specific manner, together with co-stimulatory and MHC molecules, as in antigen-presenting cell (APC) presentation [28]. mRNAs of some antigens, for example of the central nervous system (CNS), are also expressed in thymic cortical epithelial cells (cTECs) or dendritic cells (DCs). Ectopic gene expression is also demonstrable in hemopoietic cells and during certain stages of spermatogenesis. Promiscuous gene expression seems to be a basal feature of all TECs, although it is most pronounced in mTECs where it appears to be functional to the thymic representation of peripheral self [29].

mTECs show the highest level of autoimmune regulator (AIRE), a nuclear transcription factor whose gene is located on chromosome 21q22.3. A defect in the AIRE gene is responsible for a rare autosomal recessive disorder known as polyendocrinopathy syndrome type I (APS1) or autoimmune polyendocrinopathy-candidiasis-ectodermal dystrophy (APECED). This disease is characterized by organ-specific autoimmunity, affecting endocrine organs (parathyroid and adrenal glands), the skin and gastrointestinal tract, with different penetrance $[30,31]$. A large number of APS1 patients are homozygous for several mutations, without any functional AIRE transcripts. Heterozygous patients present a reduced level of functional factor and only a subclinical phenotype. The hypothesis that AIRE drives gene expression in mTECs is supported by studies showing a downregulation of the level of organ-specific gene transcripts in AIRE-deficient mTECs compared with the wild type. In the absence of AIRE, the number of epitopes expressed on mTECs is reduced, with the consequent failure in negative selection of developing autoreactive thymocytes $[31,32]$. However, AIRE-deficient mTECs maintain normal levels of other ectopically expressed self-proteins, such as C-reactive protein or glutamic-acid decarboxylase. Thus, AIRE seems to regulate only a subset of transcripts, while other AIRE-like proteins might drive the expression of other genes encoding for ectopically expressed proteins.

A further mechanism, in addition to AIRE, which may explain promiscuous gene expression is represented by epigenetic regulation of DNA methylation [29]. As previously seen, DNA methylation induces a downregulation of gene transcription, thus a reduction in methylation levels may contribute to ectopic gene expression. This phenomenon seems to occur in the testis where, during male gametogenesis, transient DNA demethylation is ob- served. Another indication is the observation that melanoma antigens, known to be strictly regulated by DNA methylation, are strongly expressed in the testis [29].

Epigenetic regulation of DNA methylation has been considered a potential mechanism also operating in the thymus. It seems to play a critical role in a model proposed to explain promiscuous gene expression in mTECs, the terminal differentiation model. In this model, during the differentiation process, $\mathrm{mTECs}$ progressively acquire the ability to express an increasing level of promiscuous genes as a result of the epigenetic regulation of DNA methylation. The process culminates with the generation of terminally mature cells, characterized by high numbers of co-stimulatory molecules (e.g. MHC II class or CD80) and transcriptional factors (e.g. AIRE) [33].

A second potential model, the developmental model, proposes instead that mTECs derive from an unique multipotent precursor, which progressively differentiates and losses his capacity to activate several transcriptional programs, with consequent gene silencing. Thus immature mTECs may express a higher number of promiscuous genes compared to mature mTECs, and this may account for the molecular mosaic of peripheral self-antigens within the thymus [34].

Finally, regardless of the model operating, during the mTEC differentiation process, a modification of gene transcription occurs which results in ectopic gene expression. This grants the central representation of peripheral self and critically contributes to tolerance. AIRE, epigenetic mechanisms and probably other still unknown events may contribute, in a complement or independent way, to the full extent of promiscuous gene expression.

\section{Take-Home Message}

- The expression of a broad spectrum of peripheral selfantigens is a basal feature of TECs, although most evident in mTEC, where it is fundamental for the establishment of central tolerance. An impairment in promiscuous gene expression may result in autoimmunity.

- The transcriptional factor, AIRE, plays a critical role in central tolerance. APECED is due to mutations in the gene encoding AIRE and in Aire-/- mice the mTEC expression of promiscuous genes is reduced or absent with the alteration of negative selection. However, the expression of many peripheral antigens is not affected in Aire-/- mice, so other mechanisms must be involved in central tolerance. 
- Two alternative models have been proposed to explain promiscuous gene expression in mTECs: the terminal differentiation model and the developmental model. In the first model, during the differentiation process, mTECs progressively acquire the ability to express an increasing level of promiscuous genes, as result of epigenetic regulation of DNA methylation. In the second model, mTECs differentiate from a unique multipotent precursor, which progressively silences its transcriptional programs and expresses a restricted profile of promiscuous genes.

\section{Infections and Autoimmunity}

A critical role for environmental factors in inducing autoimmune diseases is suggested by discordant incidence of several autoimmune diseases, such as T1D and SLE, in monozygotic twins. Epidemiological considerations support this hypothesis, such as geographic and seasonal differences in T1D incidence $[35,36]$.

Infectious agents represent a potent stimulus for the immune system and may contribute to select autoreactive $\mathrm{T}$ cells in susceptible subjects. Several associations between infections and autoimmune diseases, such as ankylosing spondylitis, multiple sclerosis and T1D, have been shown, but a pathogenic correlation between these two events has not been found.

Molecular mimicry, a potentially pathogenic mechanism, implies a cross-reactivity between microbial and self determinants (fig. 4). Random assembly of antibody variable $(V)$, diversity $(D)$ and joining $(J)$ gene segments generates a large repertoire of antigen receptors which recognize foreign and autoantigens.

Degenerate recognition of $\mathrm{T}$ cell epitopes may also contribute to cross-reactivity. Indeed, originally the TCR was thought to be reactive only against a specific peptide, while now it is known that it may recognize more antigens with a certain structural similarity. This intrinsic cross-reactivity is functional to a complete coverage of pathogen-derived antigens. Several factors may contribute to degenerate recognition. The level of surface TCRs may play a critical role. In the presence of high levels of TCRs also weak ligands are able to induce $\mathrm{T}$ cell responses. The peptide-MHC affinity is another important parameter to explain cross-reactivity. Instable peptideMHC complexes may not be able to determine negative selection in the thymus, allowing the escape of autoreactive $\mathrm{T}$ cells, but the same low-affinity complexes may induce $\mathrm{T}$ cell proliferation in the periphery. Finally, $\mathrm{T}$ cells bearing two distinct TCRs, with different specificities, represent another aspect of $\mathrm{T}$ cell cross-reactivity. The first pathogen-specific TCR may be positively selected, allowing the second autoreactive TCR to escape negative selection and reach the periphery. This phenomenon has been described for dual receptor T cells bearing an insulin-specific TCR $[37,38]$.

Thus, there are several mechanisms which allow lymphocytes activated by infectious agents to interact with self determinants. However, cross-reactivity is not sufficient to mount an autoimmune response, above all because a low-affinity epitope is often not sufficient to active the cross-reactive $\mathrm{T}$ cell, and additional factors are needed.

Transgenic mice are useful models to investigate possible operating mechanisms. The hypothesis is that, before the onset of T1D, a certain level of self or viral antigens might be strictly expressed in the pancreatic $\beta$ cells. The initiation of an immune response may occur when a triggering event, an environmental factor or a microbial infection, determines the exposition of cross-reacting antigens. This induces a consequent activation of the cellular immunity and the progression of the disease after a lag period, as suggested by murine models $[39,40]$. Transgenic mice expressing lymphocytic choriomeningitis virus (LCMV) epitopes on the $\beta$ cell surface do not develop diabetes spontaneously. T1D is found in a high percentage only after LCMV infection, since T cells activated by viral infection recognize identical epitopes on the $\beta$ cell surface [41]. The high percentage of T1D is due to the molecular identity between viral and cellular epitopes. If a low-affinity epitope was used, diabetes does not occur. Therefore, molecular mimicry is poorly effective in generating autoimmune diabetes de novo in healthy individuals, with no preexisting islet damage, and in the presence of low-affinity epitopes [41-43].

Bystander activation is a non-specific mechanism, correlated to the inflammatory response. In the presence of an inflammatory process, several chemokines and cytokines are produced and they may attract and active different 'bystander' cells, in an antigen-independent way. Bystander activation may also elicitate a response by anergic cells or induce the proliferation of low-affinity autoreactive cells, which avoided thymic negative selection. For example, Coxsackie virus infection and the consequent inflammation may accelerate diabetes onset in NOD mice in a pre-diabetic phase, enhancing the expansion of autoreactive $\mathrm{T}$ cells in a nonspecific way $[44,45]$.

Epitope spreading is an alternative mechanism, potentially explaining autoimmunity onset. After a cell dam- 
age, self-antigens are exposed and they may become new targets for immune responses. The specific immune response, initially limited to a region of strong reactivity, can extend to include other epitopes within the same protein (intramolecular epitope spreading) or other polypeptides within the same antigenic complex (intermolecular epitope spreading). The physiological role of spreading may be to allow a more rapid and intense secondary response with a longer cellular memory $[2,46]$.

It has been demonstrated in SLE that the humoral response increases in complexity over a period of time, becoming able to recognize a larger spectrum of autoantigens. A typical example of this scenario is offered by the recurrence of certain autoantibodies against different components of small nuclear ribonucleoprotein (snRNP). Thus, anti-RNP autoantibodies are often associated with anti-Smith autoantibodies and anti-Ro autoantibodies with anti-La autoantibodies. A possible interesting explanation is that the immune response to self-antigens in SLE is induced by foreign peptides through a mechanism of molecular mimicry and then epitope spreading accounts for the autoantibody diversification. As for other processes, epitope spreading probably cannot explain it all, but it may elucidate mechanisms underlying autoantibody generation [47].

However, molecular mimicry, bystander activation and epitope spreading lead or contribute to autoimmune diseases only in susceptible individuals, suggesting a complementary role for genetic and environmental factors.

\section{Apoptosis: The Model of the Autoimmune Lymphoproliferative Disease}

An impaired generation or clearance of apoptotic cells results in autoantigen production, while an alteration in apoptosis mechanisms may induce resistance to the normal process of cell death.

A paradigmatic example is offered by the autoimmune lymphoproliferative syndrome (ALPS) due to mutations in Fas sequences and the consequent alteration in Fas-induced apoptosis. Fas (Apo-1 or CD95) is a cell-surface receptor, belonging to the tumor necrosis factor receptor (TNFR) superfamily. The interaction of the Fas-Fas ligand (FasL) induces a series of events including activation of the caspase cascade and mitochondria alterations and results in cell apoptosis. T cell activation upregulates Fas, but the high levels of anti-apoptotic molecules (Bcl-2, FLIP) block apoptosis. When these molecules are down- regulated, the apoptosis cascade is induced in activated lymphocytes and the immune response is switched off. The result is a limitation of immune responses and $\mathrm{T}$ lymphocyte pool size, with a reduced risk of cross-reaction to autoantigens $[48,49]$.

ALPS is an inherited disease characterized by splenomegaly, lymphadenopathy, hypergammaglobulinemia (both IgG and IgA) and autoimmune events that essentially involve blood cells (i.e. thrombocytopenia, anemia, neutropenia). A typical ALPS feature is the peripheral expansion of a polyclonal subset of $\mathrm{T}$ cells, denominated by double-negative (DN) T cells. DN T cells express the typical mature cell determinants, CD 3 and $\alpha / \beta$ TCR, while they lack both CD4 and CD8 co-receptors [50, 51].

ALPS classification is based on the underlying genetic defect which determines disease severity. In ALPS type 0, a homozygous mutation in Fas sequences results in a complete abrogation of Fas transcript with a consequent severe disease [52]. In type I disease, there is a heterozygous dominant mutation in Fas (type Ia) or FasL sequences (type Ib, rare), which is associated with a partial deficiency in apoptosis signaling $[53,54]$. ALPS type II is due to a mutation in caspase 10 . ALPS type III is normally a sporadic disease with an incomplete clinical phenotype, whose genetic defect is unknown [51]. In six children with the sporadic form of ALPS type III, the presence of mosaicisms has recently been demonstrated to be associated with heterozygous Fas mutations in hematopoietic cells, which develop a selective advantage during hematopoiesis and generate a large amount of mutant cells (both lymphoid and myeloid). Some $\mathrm{T}$ cell responses (maybe targeting autoantigens) involve mutant $\mathrm{T}$ cells, which resist Fas-induced apoptosis, proliferate in a uncontrolled manner and lose their co-receptors, becoming DN T cells [51].

Alterations in Fas-induced apoptosis are present also in other autoimmune diseases, such as T1D and multiple sclerosis (MS). Fas defects are signaled in less than 30\% of these patients, but this proportion rises up to $70 \%$ in those affected by aggressive forms, for example in the presence of T1D associated with thyroiditis, juvenile rheumatoid arthritis, autoimmune hepatitis, thrombocytopenia, vitiligo or alopecia. These patients do not have a Fas gene mutation or DN T cell expansion, nor do they have lymphadenopathy or splenomegaly, and Fas resistance has been evidenced in vitro only in the presence of a low concentration of IL-2. Thus, the Fas signaling defect in these patients might be subtle and not sufficient to impair the switch-off of immune responses at high IL-2 levels, but it might affect apoptosis mechanisms during sub- 
tle chronic immune responses and contribute to autoimmune disease $[55,56]$.

Moreover, relatives of ALPS patients often display an increased frequency of tumors, especially lymphomas. Interestingly, autoimmune disease frequency is increased in the paternal line, while tumor frequency seems to be higher in the maternal line $[49,57]$.

Apoptosis constitutes a fundamental process in tissue homeostasis and in peripheral tolerance. Apoptotic fragments of epithelial cells, derived from homeostatic cellular turnover and presented by immature DCs, generate tolerance to tissue-specific autoantigens [58-61]. Hugues et al. [62] demonstrated that the induction of limited apoptosis of pancreatic $\beta$ cells before the onset of the disease prevents the development of pathogenic autoreactive $\mathrm{T}$ cells. Probably antigens derived from $\beta$ cell apoptosis are captured by immature DCs in the absence of inflammatory stimulus, with the consequent development of regulatory $\mathrm{T}$ cells and suppression of autoreactive $\mathrm{T}$ cells. The caspase cascade inhibition abolished the positive effect. In NOD mice, the homeostatic $\beta$ cell apoptosis probably fails to generate sufficient amounts of tolerogenic autoantigens [62]. Thus, not only excessive but also insufficient apoptosis may configure an autoimmune susceptibility status by reducing the tolerogenic phenomenon.

However, alterations in the generation and clearance of apoptotic cells may also represent a key factor for autoimmunity, since they are an important source of autoantigens. Insulitis is the precipitating event leading to T1D. This process is due to the activation in the pancreatic lymph nodes of specific $\mathrm{T}$ cells by mature APCs charged with antigens derived from $\beta$ cells. In the BDC2.5 TCR transgenic mouse model of autoimmune diabetes, priming of specific $\mathrm{T}$ cells occurs from 15 to 18 days of age. An interesting observation is that, immediately before this period in these mice, an increase in $\beta$ cell death occurs. This process may activate regional APCs which, loaded with $\beta$ cell antigens, migrate into pancreaticlymph nodes and prime naive diabetogenic $\mathrm{T}$ cells, initiating a series of events which lead to insulitis [63]. Evidence supports this hypothesis, for example experimental increases in $\beta$ cell death are associated with precocious activation of effector cells and acceleration of insulitis, while blockade of the caspase-dependent death pathway delays the appearance of antigen-loaded APCs in pancreatic lymph nodes [63]. Finally, in steady-state conditions, apoptosis occurring during homeostatic cellular turnover contributes to peripheral tolerance. Diversely, an excessive generation of apoptotic cells may result in autoantigen production and precipitate autoimmune events, probably in the presence of an inflammatory environment which may contribute to activate APCs.

Alterations in clearance of apoptotic cells may also favor autoimmune phenomena. Defects in $\mathrm{Clq}$ and $\mathrm{C} 4$ complement factors are correlated with an increased prevalence of autoimmune diseases such as SLE. In systemic autoimmune diseases, autoantigens are concentrated in subcellular particles (blebs) on the apoptotic cell surface, as evidenced by studies on murine models of neonatal lupus syndrome [58, 59]. C1q binds to late apoptotic cells, by interaction with apoptotic blebs, and induces complement activation and the uptake of apoptotic cells by macrophages [64-67]. C1q defects may determine an ineffective clearance with pro-inflammatory modifications, such as cytokine secretion and DC maturation $[66,67]$.

In conclusion, apoptosis may predispose to autoimmune diseases through different mechanisms (fig. 5), often associated with a genetic defect. In such conditions, pro-inflammatory events may alter the balance between tolerance and immunity and induce an autoimmune disease.

\section{References}

1 Sarvetnick N, Ohashi PS: Autoimmunity. Curr Opin Immunol 2003;15:647-650.

2 Christen U, von Herrath MG: Initiation of autoimmunity. Curr Opin Immunol 2004; 16:760-768.

-3 Ohashi PS: Negative selection and autoimmunity. Curr Opin Immunol 2003;15:668676.

4 Raman K, Mohan C: Genetic underpinnings of autoimmunity-lessons from studies in arthritis, diabetes, lupus and multiple sclerosis. Curr Opin Immunol 2003;15:651-659.

\footnotetext{
5 Larsen CE, Alper CA: The genetics of HLAassociated disease. Curr Opin Immunol 2004;16:660-667.

6 Shlomchik MJ, Cooke A, Weigert M: Autoimmunity. The genes and phenotypes of autoimmunity. Curr Opin Immunol 2004; 16: 738-740.

7 Germain RN: T-cell development and the CD4-CD8 lineage decision. Nature 2002;2: 309-322.

-8 Parel Y, Chizzolini C: CD4+CD8+ double positive (DP) cells in health and disease. $\mathrm{Au}-$ toimmun Rev 2004;3:215-220.

-9 Kim MS, Polychronakos C: Immunogenetics of type 1 diabetes. Horm Res 2005;64:180188.

10 Eisenbarth GS: Immunogenetics/immunopathogenesis of type 1 diabetes. Ann NY Acad Sci 2003;1005:109-118.

11 Melanitou E, Fain P, Eisenbarth GS: Genetics of type $1 \mathrm{~A}$ (immune mediated) diabetes. J Autoimmun 2003;21:93-98.
} 
$\checkmark 12$ Hamalainen AM, Knip M: Autoimmunity and familial risk of type 1 diabetes. Curr Diab Rep 2002;2:347-353.

13 Lernmark B, Elding-Larsson H, Hansson G, Lindberg B, Lynch K, Sjoblad S: Parent responses to participation in genetic screening for diabetes risk. Pediatr Diabetes 2004;5: 174-181.

14 Alper CA, Dubey DP, Awdeh Z: A simple estimate of the general population frequency of the MHC susceptibility gene for autoimmune polygenic disease. Exp Clin Immunogenet 2000;17:138-147.

15 Alper CA, Awdeh Z: Incomplete penetrance of MHC susceptibility genes: prospective analysis of polygenic MHC-determined traits. Tissue Antigens 2000;56:199-206.

-16 Kristiansen OP, Larsen ZM, Pociot F: CTLA4 in autoimmune diseases - a general susceptibility gene to autoimmunity? Genes Immun 2000;1:170-184.

17 Ueda H, Howson JM, Esposito L, Heward J, Snook H, Chamberlain G, Rainbow DB, Hunter KM, Smith AN, Di Genova G, Herr MH, Dahlman I, Payne F, Smyth D, Lowe C, Twells RC, Howlett S, Healy B, Nutland S, Rance HE, Everett V, Smink LJ, Lam AC, Cordell HJ, Walker NM, Bordin C, Hulme J, Motzo C, Cucca F, Hess JF, Metzker ML, Rogers J, Gregory S, Allahabadia A, Nithiyananthan R, Tuomilehto-WolfE, Tuomilehto J, Bingley P, Gillespie KM, Undlien DE, Ronningen KS, Guja C, Ionescu-Tirgoviste C, Savage DA, Maxwell AP, Carson DJ, Patterson CC, Franklyn JA, Clayton DG, Peterson LB, Wicker LS, Todd JA, Gough SC: Association of the T-cell regulatory gene CTLA4 with the susceptibility to autoimmune disease. Nature 2003;423:506-511.

18 Chistiakov DA, Turakulov RI: CTLA-4 and its role in autoimmune thyroid disease. J Mol Endocrinol 2003;31:21-36.

19 Januchowski R, Prokop J, Jagodzinski P: Role of epigenetic DNA alterations in the pathogenesis of systemic lupus erythematosus. J Appl Genet 2004;45:237-248.

-20 Singal R, Ginder GD: DNA methylation. Blood 1999;93:4059-4070.

-21 Yung R, Powers D, Johnson K, et al: Mechanisms of drug-induced lupus. T cells overexpressing lymphocyte function-associated antigen 1 become autoreactive and cause a lupus-like disease in syngenic recipients. J Clin Invest 1996;97:2866-2871.

-22 Gantner F, Hermann P, Nakashima K, Matsukawa S, Sakai K, Bacon KB: CD40-dependent and -independent activation of human tonsil B cells by $\mathrm{CpG}$ oligodeoxynucleotides. Eur J Immunol 2003;33:1576-1585.

-23 Lavie L, Kitova M, Maldener E, Meese E, Mayer J: CpG methylation directly regulates transcriptional activity of the human endogenous retrovirus family HERV-K(HML-2). J Virol 2005;79:876-883.
24 Okahara G, Matsubara S, Oda T, Sugimoto J, Jinno Y, Kanaya F: Expression analyses of human endogenous retroviruses (HERVs): tissue-specific and developmental stage-dependent expression of HERVs. Genomics 2004;84:982-990.

25 Ohashi P: Negative selection and autoimmunity. Curr Opin Immunol 2003;15:668-676.

26 Heath VL, Moore NC, Parnell SM, Mason DW: Intrathymic expression of genes involved in organ specific autoimmune disease. J Autoimmun 1998;11:309-318.

27 Thébault-Baumont K, Dubois-Laforgue D, Krief P, Briand JP, Halbout P, Vallon-Geoffroy K, Morin J, Laloux V, Lehuen A, Carel JC: Acceleration of type 1 diabetes mellitus in pro-insulin 2-deficient NOD mice. J Clin Invest 2003;111:851-857.

28 Derbinski J, Schulte A, Kyewski B, Klein L: Promiscuous gene expression in medullary epithelial cells mirrors the peripheral self. Nat Immunol 2001;2:1032-1039.

29 Gotter J, Brors B, Hergenhahn H, Kyewski B: Medullary epithelial cells of the human thymus express a highly diverse selection of tissue-specific genes colocalized in chromosomal clusters. J Exp Med 2004;199: 155-166.

30 Su MA, Anderson MS: Aire: an update. Curr Opin Immunol 2004; 16:746-752.

31 Bjorses P, Halonen M, Palvimo JJ, Kolmer M, Aaltonen J, Ellonen P, Perheentupa J, Ulmanen I, Peltonen L: Mutations in the AIRE gene: effects on subcellular location and transactivation function of the autoimmune polyendocrinopathy-candidiasis-ectodermal dystrophy protein. Am J Hum Genet 2000;66:378-392.

- 32 Liston A, Lesage S, Wilson J, Peltonen L, Goodnow CC: Aire regulates negative selection of organ-specific T cells. Nat Immunol 2003;4:350-354

33 Derbinski J, Gabler J, Brors B, Tierling S, Jonnakuty S, Hergenhahn M, Peltonen L, Walter J, Kyewski B: Promiscuous gene expression in thymic epithelial cells is regulated at multiple levels. J Exp Med 2005;1:33-45.

34 Gillard GO, Farr AG: Contrasting models of promiscuous gene expression by thymic epithelium. J Exp Med 2005;1:15-19.

35 Christen U, von Herrath MG: Induction, acceleration or prevention of autoimmunity by molecular mimicry. Mol Immunol 2004;40: 1113-1120.

-36 Oldstone MB: Molecular mimicry and immune-mediated diseases. FASEB J 1998;12: 1255-1265.

- 37 Kersh GJ, Allen PM: Structural basis for T cell recognition of altered peptide ligands: a single $\mathrm{T}$ cell receptor can productively recognize a large continuum of related ligands. J Exp Med 1996;184:1259-1268.

-38 Ford ML, Evavold BD: Degenerate recognition of T cell epitopes: impact of T cells receptor reserve and stability of peptide: $\mathrm{MHC}$ complexes. Mol Immunol 2004;40:10191025 .
39 Juedes AE, Rodrigo E, Togher L, Glimcher LH, von Herrath MG: T-bet controls autoaggressive CD8 lymphocyte responses in type 1 diabetes. J Exp Med 2004;199:1153-1162.

40 Christen U, Edelmann KH, McGavern DB, Wolfe T, Coon B, Teague MK, Miller SD, Oldstone MB, von Herrath MG: A viral epitope that mimics a self antigen can accelerate but not initiate autoimmune diabetes. J Clin Invest 2004;114:290-298.

41 von Herrath MG, Dockter J, Oldstone MB: How virus induces a rapid or slow onset insulin-dependent diabetes mellitus in a transgenic model. Immunity 1994;1:231-242.

42 Panoutsakopoulou V: Analysis of the relationship between viral infection and autoimmune disease. Immunity 2001;15:137-147.

43 Chen HD, Fraire AE, Joris I, Welsh RM, Selin LK: Specific history of heterologous virus infections determines anti-viral immunity and immunopathology in the lung. Am J Pathol 2003;163:1341-1355.

-44 Horwitz MS, Bradley LM, Harbertson J, Krahl T, Lee J, Sarvetnick N: Diabetes induced by Coxsackie virus: initiation by bystander damage and not molecular mimicry. Nat Med 1998;4:781-785.

45 Mena I, Fischer C, Gebhard JR, Perry CM, Harkins S, Whitton JL: Coxsackie virus infection of the pancreas: evaluation of receptor expression, pathogenesis, and immunopathology. Virology 2000;271:276-288.

46 Carl PL, Temple BR, Cohen PL: Most nuclear systemic autoantigens are extremely disordered proteins: implications for the etiology of systemic autoimmunity. Arthritis Res Ther 2005;7:1360-1374.

47 Deshmukh US, Kannapell CC, Fu SM: Immune responses to small nuclear ribonuceloproteins: antigen-dependent distinct $\mathrm{B}$ cell epitope spreading patterns in mice immunized with recombinant polypeptides of small nuclear ribonucleoproteins. J Immunol 2002;169:5326-5332.

48 Nagata S, Suda T: Fas and Fas ligand: lpr and gld mutations. Immunol Today 1995;16:3943.

49 Dianzani U, Chiocchetti A, Ramenghi U: Role of inherited defects decreasing Fas function in autoimmunity. Life Sci 2003;72: 2803-2824.

50 Fisher GH, Rosenberg FJ, Straus SE, et al: Dominant interfering Fas gene mutations impair apoptosis in a human autoimmune lymphoproliferative syndrome. Cell 1995; 81:935-946.

-51 Holzelova E, Vonarbourg C, Stolzenberg MC, Arkwright PD, Selz F, Prieru AM, Blanche S, Bartunkova J, Vilmer E, Fischer A, Le Deist F, Rieux-Lacaut F: Autoimmune lymphoproliferative syndrome with somatic Fas mutations. N Engl J Med 2004;351:1409_ 1418. 
-52 van der Burg M, de Groot R, Comans-Bitter $\mathrm{WM}$, et al: Autoimmune lymphoproliferative syndrome (ALPS) in a child from consanguineous parents: a dominant or recessive disease? Pediatr Res 2000;47:336-343.

$\checkmark 53$ Jackson CE, Fischer RE, Hsu AP: Autoimmune lymphoproliferative syndrome with defective Fas: genotype influences penetrance. Am J Hum Genet 1999;64:10021014.

-54 Rieux-Laucat F, Blachere S, Danielan S, et al: Lymphoproliferative syndrome with autoimmunity: a possible genetic basis for dominant expression of the clinical manifestations. Blood 1999;94:2575-2582.

-55 Comi C, Leone M, Bonissoni S, De Franco S, Bottarel F, Mezzatesta C, Chiocchetti A, Perla F, Monaco F, Dianzani U: Detective T cells Fas function in patient with multiple sclerosis. Neurology 2000;55:921-927.

56 De Franco S, Bonissoni S, Cerutti F, Bona G, Bottarel F, Cadario F, Brusco A, Loffredo G, Rabbonì I, Corrias A, Pignata C, Ramenghi U, Dianzani U: Defective function of Fas in patients with type 1 diabetes associated with other autoimmune dieseas. Diabetes 2001; 50:483-488.
57 Ramenghi U, Bonissoni S, Migliaretti G, et al: Deficiency of the Fas apoptosis pathway without Fas gene mutations is a familial trait predisposing to development of autoimmune diseases and cancer. Blood 2000;95: 3176-3182.

58 Cline AM, Radic MZ: Apoptosis, subcellular particles, and autoimmunity. Clin Immunol 2004;112:175-182.

59 Cocca BA, Cline AM, Radic MZ: Blebs and apoptotic bodies are B cell autoantigens. J Immunol 2002;169:159-166.

60 Tran HB, Macardle PJ, Hiscock J: Anti-La/ SSB antibodies transported across the placenta bind apoptotic cells in fetal organs targeted in neonatal lupus. Arthritis Rheum 2002;46:1572-1579.

61 Huang FP, Platt N, Wykes M, Major JR, Powell TJ, Jenkins CD, MacPherson GG: A discrete subpopulation of dendritic cells transports apoptotic intestinal epithelial cells to T-cell areas of mesenteric lymph nodes. J Exp Med 2000;191:435-444.
62 Hugues S, Mougneau E, Ferlin W, Jeske D, Hofman P, Homann D, Beaudoin L, Schrike C, Von Herrat M, Lehuen A, Glaichenhaus $\mathrm{N}$ : Tolerance to islet antigens and prevention from diabetes induced by limited apoptosis of pancreatic $\beta$ cells. Immunity 2002;16: 169-181.

63 Turley S, Poirot L, Hattori M, Benoist C, Mathis D: Physiological $\beta$ cell death triggers priming of self-reactive $T$ cells by dendritic cells in a type 1 diabetes model. J Exp Med 2003;198:1527-1537.

64 Morgan BP, Walport MJ: Complement deficiency and disease. Immunol Today 1991;12: 301-306.

65 Botto M, Dell'Agnola C, Bygrave AE: Homozygous C1q deficiency causes glomerulonephritis associated with multiple apoptotic bodies. Nat Genet 1998;19:56-59.

66 Taylor PR, Carugati A, Fadok VA: A hierarchical role for classical pathway complement proteins in the clearance of apoptotic cells in vivo. J Exp med 2000;192:359-366.

67 Nauta AJ, Trouw LA, Daha MR, et al: Direct binding of $\mathrm{Clq}$ to apoptotic cells and cell blebs induces complement activation. Eur J Immunol 2002;32:1726-1736. 